\title{
The mechanical performance of 3D printed hierarchical honeycombs using carbon fiber and carbon nanotube reinforced acrylonitrile butadiene styrene filaments
}

\author{
Michel Theodor Mansour ${ }^{1, *}$, Konstantinos Tsongas ${ }^{2}$, and Dimitris Tzetzis ${ }^{3}$ \\ ${ }^{1}$ Technical University of Crete, School of Production Engineering and Management, Chania, Greece \\ ${ }^{2}$ Aristotle University of Thessaloniki, Department of Mechanical Engineering, Thessaloniki, Greece \\ ${ }^{3}$ International Hellenic University, School of Science and Technology, Thermi, Greece
}

\begin{abstract}
The aim of this paper is to design hierarchical honeycombs as well as manufacturing such structures with a commercial 3D Printer using Fused Filament Fabrication (FFF) technique. The materials under study are commercial filaments such as acrylonitrile butadiene styrene (ABS), acrylonitrile butadiene styrene/carbon fibers $(\mathrm{ABS} / \mathrm{CF})$ and acrylonitrile butadiene styrene/carbon nanotubes (ABS/CNTs). The fabricated hierarchical honeycombs were examined by compression tests in order to evaluate the mechanical behaviour of such honeycomb 3D printed structures. The compression behaviour of the hierarchical honeycombs was assessed also with finite element analysis (FEA) and at the end there was a comparison with the experimental findings. The results reveal that the $2^{\text {nd }}$ order hierarchy presented an increase both in stiffness and strength in comparison with the $0^{\text {th }}$ and the $1^{\text {st }}$ hierarchies which make such designs a suitable for structures require such properties. Also, the results reveal that $\mathrm{ABS} /$ carbon fiber constructs outperform the other materials under study.
\end{abstract}

\section{Introduction}

In the last few years, 3D Printing had a tremendous impact on the manufacturing industry globally. This satisfactory outcome could be ascribed mainly to its exceptional capability to directly fabricate complex parts without special tools and at the same time to the decrease of material waste, time and cost of fabrication $[1,2]$. It is widely acknowledged that there is a shortage of printable materials on the market nowadays for special applications due to their inferior mechanical performance, so it is very essential to develop printable materials with superior mechanical behaviour [1-3]. For that reason, composite filaments have been regarded as promising 3D-printing feedstocks due to their compelling combination properties of mechanical robustness, lightweight and versatility. Combining such materials with

\footnotetext{
* Corresponding author: mmansour@isc.tuc.gr
} 
honeycomb structures, it is possible to make a lighter and at the same time stronger structures possibly with high-energy absorption capability and good crashworthiness behaviour [4].

In the current work, the aim was to present the design and fabrication of hierarchical honeycombs structures with three different printing materials but with the same polymer matrix. The materials used were an acrylonitrile butadiene styrene (ABS) plastic filament as well as composites such as acrylonitrile butadiene styrene with carbon fibers (ABS/CF) and acrylonitrile butadiene styrene with carbon nanotubes (ABS/CNTs). A computer generated algorithm was developed in order to create parametrically each hierarchical honeycomb structure, giving as input dimensions of the honeycomb such as width, number of hexagons horizontally and vertically and the hierarchy of the structure. Furthermore, the mechanical behaviour of these hierarchical honeycomb structures was evaluated by uniaxial compression tests. Finally, the experimental results were compared with predictions from finite element analysis (FEA), in order to understand these honeycomb structures and utilizing them for real life structures and components.

\section{Design of hierarchical honeycombs}

In the current work the vertices of a regular hexagonal lattice structure are replaced with smaller hexagons to achieve a shape with one level of hierarchy. Simultaneously the wall thickness is diminished so as to maintain the overall density. It is anticipated that higher hierarchies will demonstrate stiffness superior to that of its regular hexagonal counterpart of equal relative density. Fractal appearing honeycombs can be achieved by such approach with higher orders of structural hierarchy, if this replacement procedure for three-edge vertices is repeated. Figure 1 (a) shows the regular, $1^{\text {st }}$ and $2^{\text {nd }}$ hierarchies of the hexagonal honeycomb cells. According to A. Ajdari et. al. [5] for each level of hierarchy the structural organization of the honeycomb can be defined as the ratio of the introduced hexagonal edge length (b for the $1^{\text {st }}$ order hierarchy and $\mathrm{c}$ for the $2^{\text {nd }}$ order hierarchy), to the original hexagon's edge length, (a), as described in Figure 1 (a) i.e., $\gamma_{1}=b / a$ and $\gamma_{2}=c / a$. For a honeycomb with $1^{\text {st }}$ order hierarchy, $0 \leq \mathrm{b} \leq \mathrm{a} / 2$ and thus, $0 \leq \gamma_{1} \leq 0.5$, where $\gamma_{1}=0$ denotes the regular honeycomb structure. For a honeycomb with $2^{\text {nd }}$ order hierarchy, there are two geometrical constraints, $0 \leq \mathrm{c} \leq \mathrm{b}$ and $\mathrm{c} \leq \mathrm{a} / 2-\mathrm{b}$. In terms of the ratio parameters, the constraints are $0 \leq \gamma_{2} \leq \gamma_{1}$ if $\gamma_{1} \geq 0.25$ and $0 \leq \gamma_{2} \leq\left(0.5-\gamma_{1}\right)$ if $0.25 \leq \gamma_{1} \leq 0.5$. The dimensionless relative density, can be given in terms of t/a:

$$
\rho=\frac{2}{\sqrt{3}} \times\left(1+2 \gamma_{1}+6 \gamma_{2}\right) \times \frac{t}{a}
$$

where $t$ is the thickness of the cell walls, from which the special cases of $\gamma_{2}, \gamma_{1}=0$ can be read off immediately. For regular honeycomb, $\rho=\frac{2}{\sqrt{3}} \times \frac{t}{a}$ and for honeycomb with first order hierarchy, $\rho=\frac{2}{\sqrt{3}} \times\left(1+2 \gamma_{1}\right) \times \frac{t}{a}$ and finally for honeycomb with second order hierarchy, $\rho=\frac{2}{\sqrt{3}} \times\left(1+2 \gamma_{1}+6 \gamma_{2}\right) \times \frac{t}{a}$. This relation clearly shows that $\mathrm{t} / \mathrm{a}$ must be reduced to maintain fixed relative density as $\gamma_{1}, \gamma_{2}$ are increased.

\section{Experiments}

\subsection{Manufacturing of 3D printed hierarchical honeycombs}

The hierarchical honeycomb structures were fabricated using a commercial open source Ultimaker 2+3D Printer through FFF printing method with a $3 \mathrm{~mm}$ extrusion nozzle and also using Cura software for fine-tuning the honeycomb specimens. Moreover, a nozzle extrusion 
temperature of $240^{\circ} \mathrm{C}$ was used with a build-plate temperature of $100^{\circ} \mathrm{C}$ and also a printing speed of $35 \mathrm{~mm} / \mathrm{s}$. These are the very basic printing parameters that were used on the hierarchical honeycomb structures under investigation. The deposition speed was kept constant in order to avoid any variabilities in the 3D printed constructs. Additionally, all specimens were 3D printed in room temperature conditions without humidity control. Commercial Acrylonitrile Butadiene Styrene (ABS), acrylonitrile butadiene styrene reinforced with $15 \%$ carbon fibers $(\mathrm{ABS} / \mathrm{CF})$ and finally acrylonitrile butadiene styrene reinforced with carbon nanotubes (ABS/CNTs) were selected as the printing materials for the fabrication of the hierarchical honeycomb structures in this paper due to their good mechanical behaviour. The weight percentage of the CNTs was proprietary figure, not disclosed from the manufacturer. In addition, all the specimens had relative density of $\rho=0.12$. The original hexagon's edge length was $a=20 \mathrm{~mm}$ and also the thickness of the cell wall was measured as $\mathrm{t}=2 \mathrm{~mm}$. Furthermore, the hierarchical honeycomb with $1^{\text {st }}$ order hierarchy had $\gamma_{1}=0.3$ and $\mathrm{t}=1.25 \mathrm{~mm}$ and at the same way the hierarchical honeycomb with $2^{\text {nd }}$ order hierarchy had $\gamma_{1}=0.3, \gamma_{2}=0.12$ and $\mathrm{t}=0.86 \mathrm{~mm}$. Also, there was a reduction in the cell wall thickness for honeycombs with the increase in the hierarchy, so as to maintain the overall relative density constant. This was kept similar to the FEA calculations.

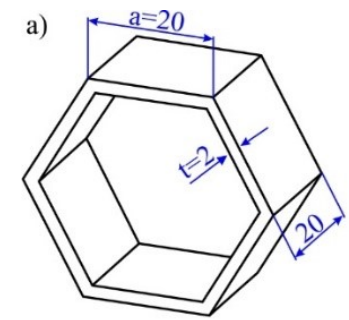

Regular

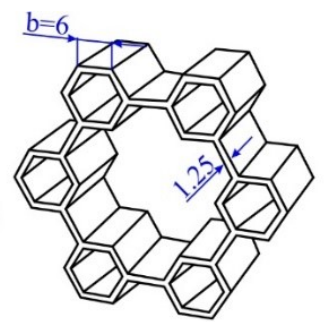

1st order hierarchy

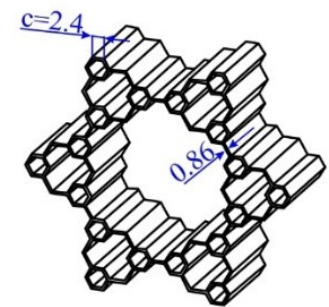

2nd order hierarchy

b)
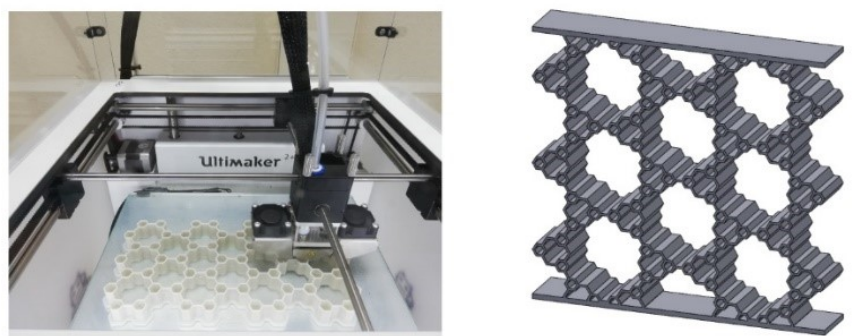

Fig. 1. Hierarchical Honeycombs: a) cell of the regular and hierarchical honeycombs and b) the printing process and the virtual model of $2^{\text {nd }}$ order hierarchy.

\subsection{Compression experiments}

The in-plane compression tests were conducted for the three different honeycomb structures by using a computer-controlled servo-hydraulic single axial test machine Testometric (UK) equipped with a $50 \mathrm{kN}$ load cell. The cross-head speed was set at $5 \mathrm{~mm} / \mathrm{min}$ and at least three specimens of the same level of hierarchy were tested. Upon testing, the load-displacement curves were calculated in order to directly be compared with the predicted ones from the finite element analysis. 


\section{Results and discussion}

The typical load-displacement curves under compressive loading for all materials under study i.e. $\mathrm{ABS}, \mathrm{ABS} / \mathrm{CNTs}$ and $\mathrm{ABS} / \mathrm{CF}$ and for each hierarchical honeycomb structure under investigation are illustrated in Figure 2. Initially, the results of ABS reveal that the curves have reached a peak compression load of $570.9 \mathrm{~N}, 586.1 \mathrm{~N}$ and $649.92 \mathrm{~N}$ for the regular honeycomb, the first order hierarchy and second order hierarchy, respectively. Similarly, such trend was observed from the results of ABS/CNTs with values of $554.16 \mathrm{~N}, 698.52 \mathrm{~N}$ and $783.8 \mathrm{~N}$. At the same way, the results for $\mathrm{ABS} / \mathrm{CF}$ shown that they have reached a peak compression load of $675.2 \mathrm{~N}, 778.7 \mathrm{~N}$ and $924.63 \mathrm{~N}$ as the hierarchy was getting higher. The highest deformation was up to $8 \mathrm{~mm}$. The results portray clearly that the second order hierarchy structure samples for ABS/CF show higher stiffness than the other materials and hierarchies. This phenomenon was expected since the effective elastic modulus is expressed as the ratio of mean stress and mean strain by the equation [5]:

where

$$
\frac{E}{E s}=(t / a)^{3} f\left(\gamma_{1}\right)
$$

$$
f\left(\gamma_{1}\right)=\sqrt{3} /\left(0.75-3.525 \gamma_{1}+3.6 \gamma_{1}{ }^{2}+2.9 \gamma_{1}{ }^{3}\right)
$$

According to A. Ajdari et al. [5], in order to find the maximum normalized elastic modulus for $1^{\text {st }}$ order of hierarchy structures with constant relative density, t/a has to be excluded from Eq. (2), using through Eq. (1) the relative density expression. This expression for $E / E_{s}$ is a $\rho^{3}$ times function of $\gamma_{1}$, while setting $\left(\partial\left(\frac{E}{E s}\right) / \partial \gamma_{1}\right)_{\rho}=0$ leads to $E_{1} / E_{s}=$ $2.97 \rho^{3}$, two times the stiffness of the zeroth level of hierarchy honeycomb structure, for which $E_{0} / E_{s}=1.5 \rho^{3}$ (regular honeycomb's effective modulus can be calculated by setting $\gamma_{1}=0$ in Eq. (2), and using Eq. (1) to eliminate t/a). For the $2^{\text {nd }}$ order hierarchical structure, a stiffness almost three point five times that of regular honeycomb was observed. The experimental load-displacement results of the honeycombs manufactured with neat ABS were in agreement with these theoretical values; 1.91 times higher stiffness for the $1^{\text {st }}$ level of hierarchy and 3.33 times for the $2^{\text {nd }}$ level, compared with the stiffness of the regular honeycomb. Considering the honeycombs manufactured using ABS/CNTs the stiffness increase was 1.92 and 3.27 for $1^{\text {st }}$ and $2^{\text {nd }}$ level of hierarchy, respectively. Such values were 2.0 and 3.50 in the case of honeycombs manufactured from $\mathrm{ABS} / \mathrm{CF}$ [6].

Overall, as the load increases and the honeycomb structure is under compression, the hierarchy cells tend to sustain deformation. This results in smaller yield displacement compared to the $2^{\text {nd }}$ order of hierarchy, $1^{\text {st }}$ order of hierarchy and regular honeycomb. Therefore, hierarchical honeycombs resist higher values of applied loads compared to the regular honeycomb system and increase the stiffness.

The effect of hierarchy on the elastic properties of 3D printed hierarchical honeycombs has been also investigated in this paper. In order to accomplish that, the compression experimental results were simulated utilizing FEA. A computational model was introduced and a static structural analysis was performed using the commercial code ANSYS. Assumptions of initial material values for the tangent moduli $E_{i}$ of the multilinear stressstrain curves of the honeycombs' were made in the FEA model. On the top plate of each honeycomb structure, a vertical displacement was applied in steps and the reaction force was obtained at the bottom, which was considered with a fixed boundary condition. The values of this vertical displacement were acquired by the experimental results shown in Figure 2. Considering the deformation, a static force was determined and compared to the experimental one. If the calculated force does not converge with the measured then the values $E_{i}$ are 
approximated and the finite element model is solved again. This procedure is repetitive until the last pair of values has converged and the loop ends. The final force-displacement values calculated from the FEA are illustrated in Figure 2, where it can be easily seen that these values converged with the experimental results. Thus, the assumptions of the multilinear material model of each hierarchy were considered accurate.

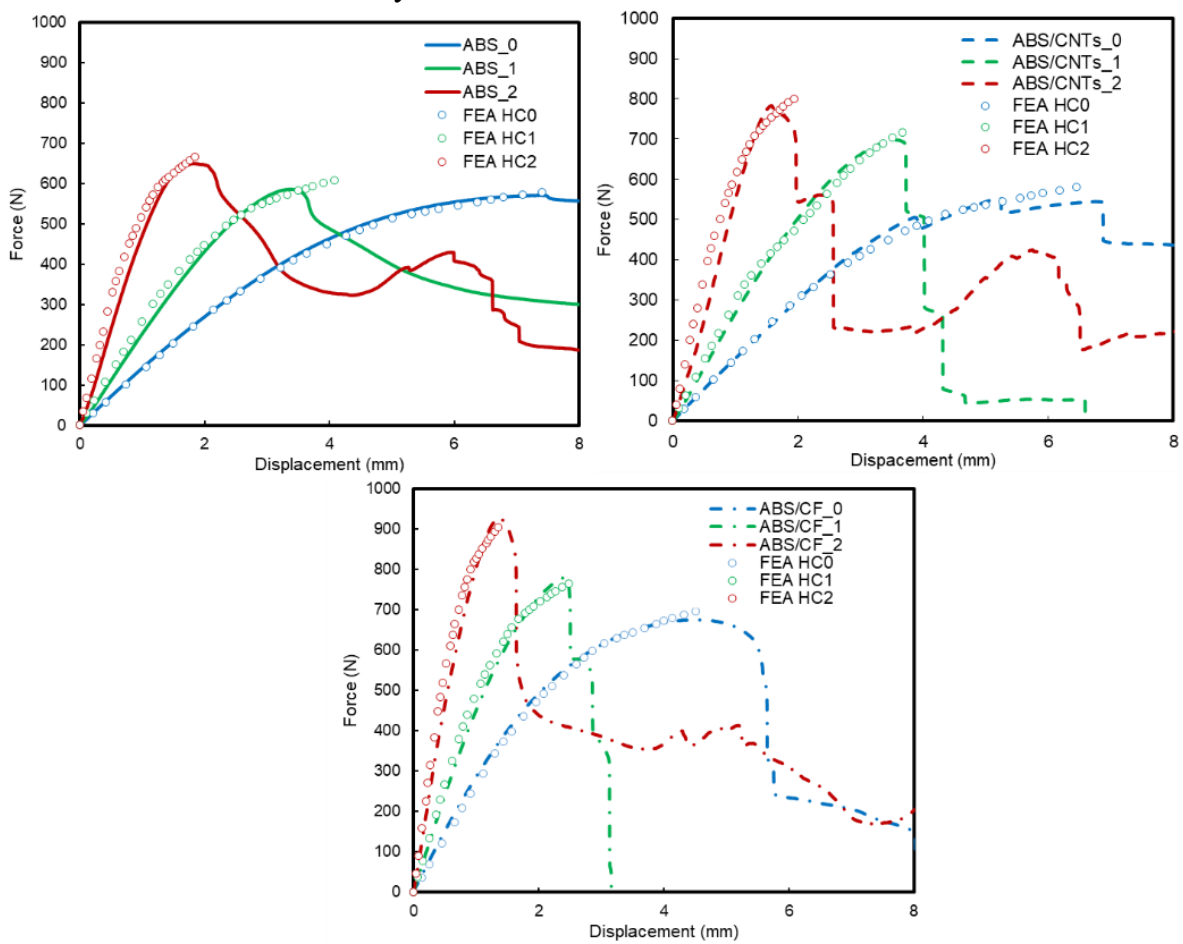

Fig. 2. Typical experimental load displacement curves and the curve-fit utilizing a FEA model of HC0, $\mathrm{HC} 1$ and $\mathrm{HC} 2$ structures for $\mathrm{ABS}, \mathrm{ABS} / \mathrm{CNT}$ s and $\mathrm{ABS} / \mathrm{CF}$ materials.

Finite Element Analyses were performed on honeycomb structures over the three levels of hierarchy, as shown in Figure 3, where the stress strain behaviour of ABS was compared with the $\mathrm{ABS} / \mathrm{CNTs}$ and $\mathrm{ABS} / \mathrm{CF}$ at different levels of hierarchy. The $E_{1}$ value (initial slope) of the elastic modulus was determined to be $2050 \mathrm{MPa}, 2350 \mathrm{MPa}$ and $3210 \mathrm{MPa}$ for ABS, $\mathrm{ABS} / \mathrm{CNTs}$ and $\mathrm{ABS} / \mathrm{CF}$, respectively. The level of hierarchy did not have any significant effect on the Young's moduli in the elastic region. First order hierarchy and second order hierarchy structures show higher strength than the regular honeycomb without compromising significantly their ultimate strain, even at higher levels of hierarchy.

\section{Conclusions}

The mechanical performance of the three different materials and the three levels of hierarchical honeycombs was evaluated through static compression tests. The results revealed increased stiffness and strength for the $\mathrm{ABS} / \mathrm{CF}$ and the 2nd order hierarchy of honeycombs in comparison with the other materials and the 0th and the 1st hierarchies, respectively. A procedure is introduced in order to extract the hierarchical honeycombs' constitutive laws based on the evaluation of uniaxial compression test results through a developed FEA simulation. The FEA model utilizes the compression test results as input data to the described model and extracts the stress-strain curves of the $3 \mathrm{D}$ printed hierarchical 
honeycombs. The procedure followed shows that the presented compression test result evaluation method is a very efficient procedure to depict the stress-strain behaviour of the $3 \mathrm{D}$ printed hierarchical honeycombs.
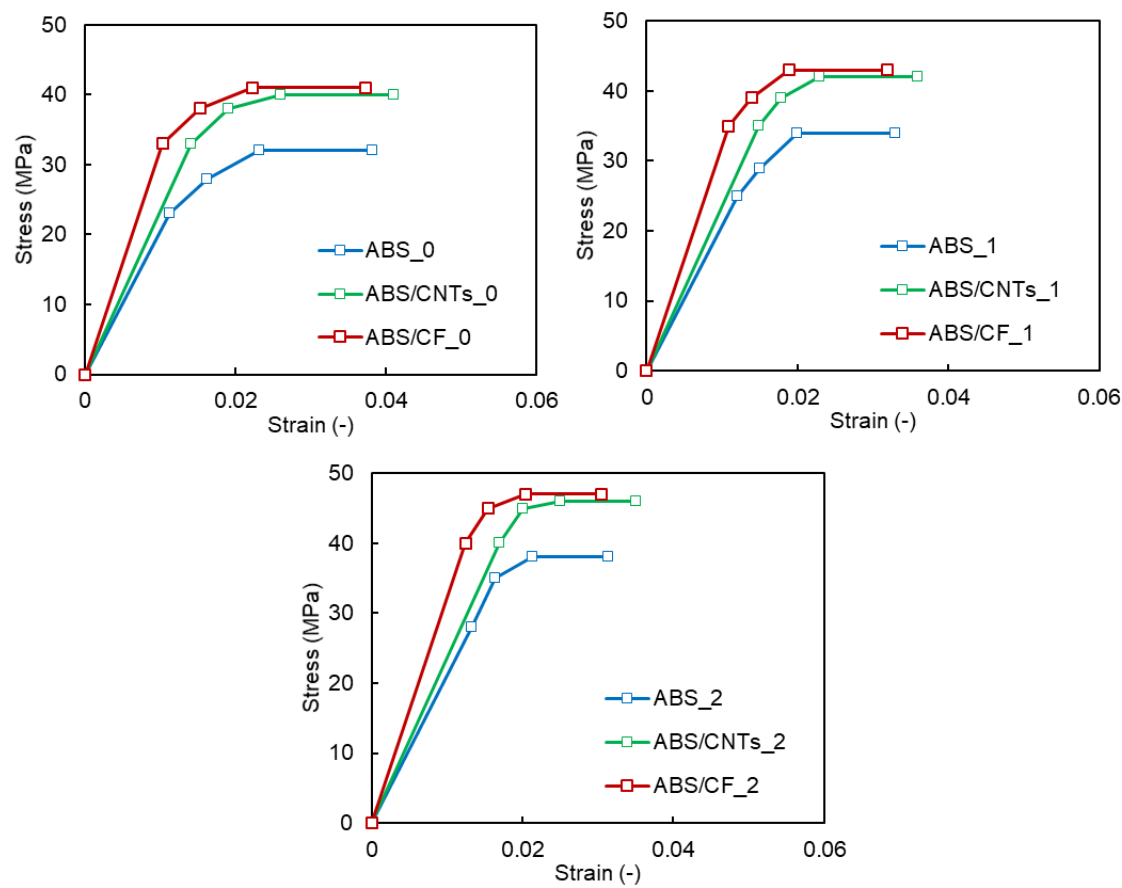

Fig. 3. Comparison among the $\mathrm{ABS}, \mathrm{ABS} / \mathrm{CNTs}$ and $\mathrm{ABS} / \mathrm{CF}$ materials for different levels of hierarchy.

This research is co-financed by Greece and the European Union (European Social Fund - ESF) through the Operational Programme «Human Resources Development, Education and Lifelong Learning» in the context of the project "Strengthening Human Resources Research Potential via Doctorate Research - 2nd Cycle" (MIS-5000432), implemented by the State Scholarships Foundation (IKY). Also, the authors would like to thank Mrs. V. Papadopoulou for her assistance with the computer generated algorithm in relation to the design of the honeycombs.

\section{References}

1. M. Mansour, K. Tsongas, D. Tzetzis, A. Antoniadis, P Polym Plast Technol Eng, 57, $1715-25$ (2018)

2. M. Mansour, K. Tsongas, D. Tzetzis, Polym Plast Technol Eng, 58, 1234-44 (2019)

3. M. T. Mansour, K. Tsongas, D. Tzetzis, A. Antoniadis, IOP Conf. Ser.: Mater. Sci. Eng., 564 (2019)

4. L. Sang, S. Han, X. Peng, X. Jian, J. Wang, Compos. Part A Appl. Sci. Manuf., 125 (2019)

5. A. Ajdari, B. H. Jahromi, J. Papadopoulos, H. Nayeb-Hashemi, A. Vaziri, Int. J. Solids Struct., 49, 1413-19 (2012)

6. R. T. L. Ferreira, I. C Amatte, T. A. Dutra, D. Bürger, Compos Part B-Eng, 124, 88-100 (2017) 